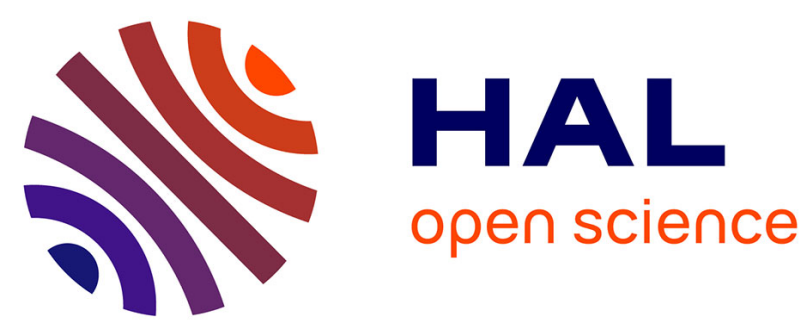

\title{
Le rempart antique de Saint-Bertrand-de-Comminges (Haute-Garonne)
}

Simon Esmonde Cleary, Jason Wood

\section{To cite this version:}

Simon Esmonde Cleary, Jason Wood. Le rempart antique de Saint-Bertrand-de-Comminges (HauteGaronne). Gallia - Archéologie de la France antique, 2006, Antiquité tardive, haut Moyen Âge et premiers temps chrétiens en Gaule méridionale (première partie) : réseau des cités, monde urbain et monde des morts, 63, pp.81-84. 10.3406/galia.2006.3286 . hal-01914297

\section{HAL Id: hal-01914297 \\ https://hal.science/hal-01914297}

Submitted on 8 Jan 2020

HAL is a multi-disciplinary open access archive for the deposit and dissemination of scientific research documents, whether they are published or not. The documents may come from teaching and research institutions in France or abroad, or from public or private research centers.
L'archive ouverte pluridisciplinaire HAL, est destinée au dépôt et à la diffusion de documents scientifiques de niveau recherche, publiés ou non, émanant des établissements d'enseignement et de recherche français ou étrangers, des laboratoires publics ou privés.

\section{(ㅇ)(1) $\$$}

Distributed under a Creative Commons Attribution - NonCommercial - NoDerivatives| 4.0 


\title{
LE REMPART ANTIQUE DE SAINT-BERTRAND-DE-COMMINGES (HAUTE-GARONNE)
}

\author{
Simon ESMONDE CLEARY et Jason WOOD
}

\begin{abstract}
Mots-clés. Étude architecturale, rempart, tour, transition entre l’Antiquité tardive et le haut Moyen Âge.
Résumé. Le programme réalisé sur le rempart de la ville haute, en 1993-2001, a consisté en une reconnaissance et en une analyse architecturale qui a permis de reconnaître la nature de la construction et de localiser une partie, bien conservée, du couronnement du rempart et des tours dans le secteur nord-est de l'enceinte. Parallèlement, une série de tranchées a révélé l'absence d'occupation avant le dernier quart du IV $V^{e}$., la construction de murs peu après le tournant des $I V^{e}$ et $V^{e}$ s., et une occupation continue jusqu'au VIII ${ }^{e}$., mais sans livrer de traces du siège de 585 décrit par Grégoire de Tours.
\end{abstract}

Key-words. Architectural analysis, walls, wall-top, tower, late Roman/early Mediaeval transition.

Abstract. The 1993-2001 project on the Roman walls of the "ville haute" comprised architectural survey and analysis which yielded an integrated understanding of the structure and also located a stretch of well-preserved late Roman wall-top and the possible locations of towers along the north-eastern part of the circuit. At the same time, a series of trenches revealed no occupation before the last quarter of the $4^{\text {th }}$ century, the construction of the walls shortly after the turn of the $4^{\text {th }}$ and $5^{\text {th }}$ centuries and occupation continuing down to the $8^{\text {th }}$ century, but with no trace of the siege of 585 described by Gregory of Tours.

Translation: Isabelle FAUDUET

Schlagwörter. Bauforschung, Befestigung, Turm, Übergang zwischen Spätantike und Frühmittelalter.

Zusammenfassung. Ein von 1993 bis 2001 durchgeführtes Projekt zur Befestigung der Oberstadt beinhaltete eine Bestandserfassung und eine bauforscherische Untersuchung, durch die die Art der Konstruktion verständlich wurde und die zur Identifizierung eines gut erhaltenen Abschnittes der Mauerbekrönung und der Türme im Nordost-Sektor des Mauerrings führte. Gleichzeitig wurde durch eine Reihe von Suchschnitten deutlich, daß vor dem letzten Viertel des vierten Jahrhunderts keine Siedlungstätigkeit bestand, daß kurz nach der Wende vom 4. zum 5. Jahrh. Mauerabschnitte errichtet wurden und daß die Besiedlung bis ins 8. Jahrh. fortdauerte. Allerdings wurden keine Spuren der bei Gregor von Tours beschriebenen Belagerung des Jahres 585 gefunden.

Übersetzung: Stefan WIRTH

Le rempart antique de Saint-Bertrand-de-Comminges (Lugdunum Convenae dans l'Antiquité), long de $885 \mathrm{~m}$, enferme un espace approximativement triangulaire de 4,4 ha au sommet du piton de calcaire qui domine les restes de la ville gallo-romaine (fig. 40). Le rempart est attesté dans le récit que fait Grégoire de Tours du siège mené en 585 par le roi Gontran contre l'usurpateur Gondovald réfugié dans les fortifications de la ville haute (Grégoire de Tours, HF, VII, 33-38). Reconnue comme antique par
R. Lizop en 1931, la muraille encore visible fut explorée, sous la forme de quatre sondages archéologiques, par B. Sapène en 1942, 1957, 1965 et 1967. Ils confirmèrent l'antiquité de l'ouvrage, sans toutefois livrer aucun élément probant de datation pour la construction de l'enceinte. De 1993 à 2001, le rempart fut étudié par une équipe britannique dans le cadre d'un projet de recherche dirigé par J. Wood (prospections et analyses architecturales) et S. Esmonde Cleary (fouilles archéologiques). 


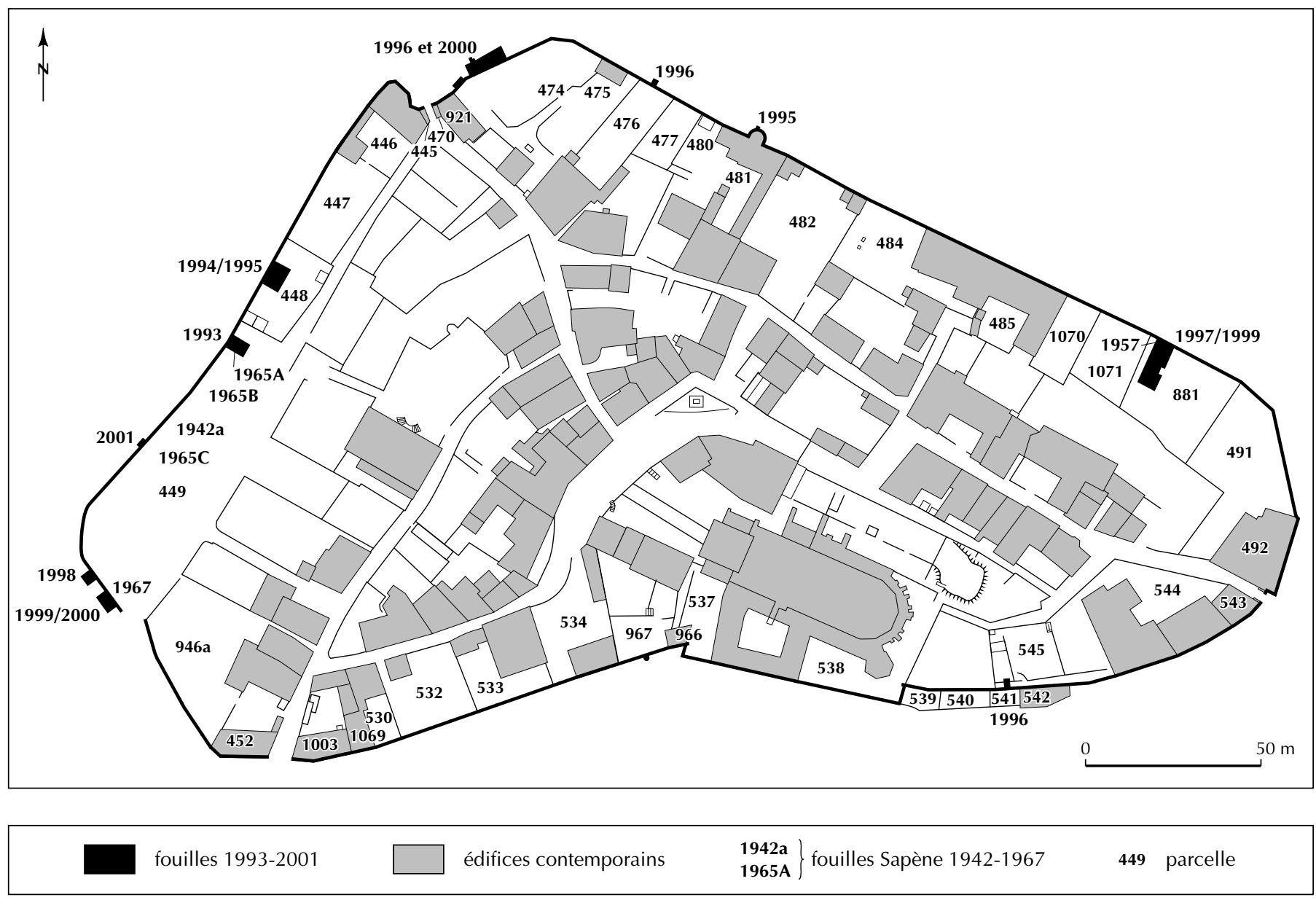

Fig. 40 - Saint-Bertrand-de-Comminges (Haute-Garonne).

Plan de la ville haute avec la localisation des fouilles archéologiques (DAO H. Buglass, université de Birmingham).

\section{PROSPECTIONS ET ANALYSES ARCHITECTURALES}

Le matériau de base utilisé dans la construction du rempart antique est composé de blocs calcaires non-taillés, qui, disposés en lits assez réguliers et liés par un mortier dur en banchées, forment le blocage interne de la muraille. Les parements sont constitués d'assises de moellons en petit appareil, rythmées par des lits de briques. L'observation des vestiges a permis de recenser dans le blocage de nombreux éléments d'architecture (fragments de corniches, fûts et chapiteaux de colonnes) et a montré le caractère très hétéroclite des éléments utilisés pour les assises: briques, tuiles, dalles. Le rempart parait donc bien avoir été édifié à partir d'une grande majorité de matériaux récupérés et remployés. La muraille se compose de quatre éléments principaux, dont subsistent aujourd'hui encore des vestiges. Se distinguent, du bas vers le haut, la fondation, le soubassement, l'élévation et le couronnement.

La fondation remplit une tranchée creusée dans l'argile naturelle, sauf à l'angle sud-ouest où elle est directement assise sur le rocher du Matacan. Elle est constituée à la base d'une couche de gros galets de la Garonne sur laquelle sont coulés des lits de pierres calcaires noyées dans du mortier. Aucun gros bloc architectural en remploi, comme les spolia qui caractérisent généralement les fortifications urbaines de l'Antiquité tardive en Gaule, n'a été identifié dans les fondations. Un retrait du parement marque la transition entre la fondation et le soubassement.

Le soubassement, élevé en lits de pierres calcaires liées au mortier, assure la transition entre la fondation et l'élévation. 


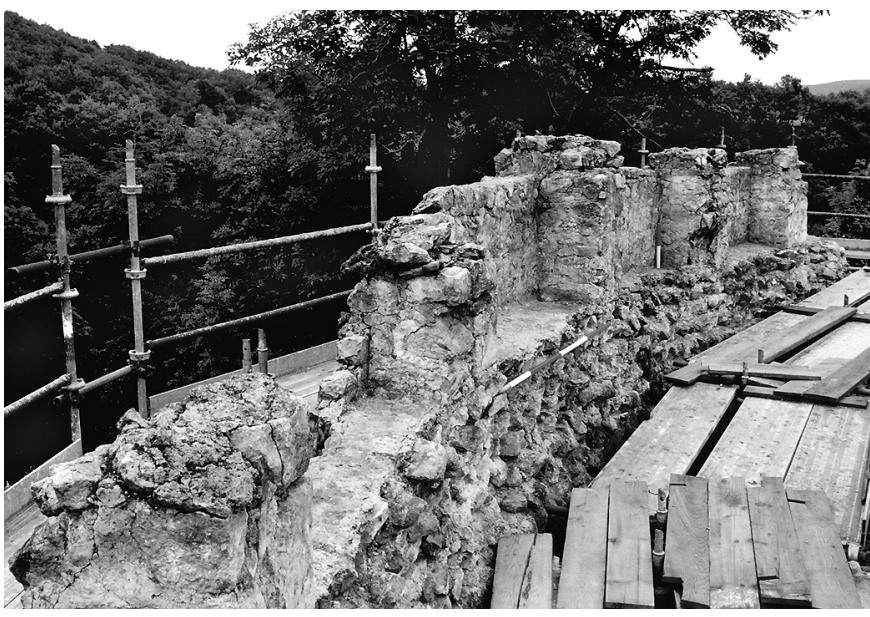

Fig. 41 - Saint-Bertrand-de-Comminges (Haute-Garonne). Vue prise de l'angle sud-ouest des vestiges du couronnement de l'enceinte (clichéJ. Wood).

L'élévation, la muraille proprement dite, en assises de moellons calcaires rythmées par des lits de briques, est large de 1,70 m. Des ressauts sont aménagés sur la face interne. Un enduit en mortier blanchâtre, revêtu de lait de chaux, a été observé dans les sondages pratiqués sur la face interne du rempart, et des traces en subsistent sur la face externe. Il recouvrait les parements, cachant ainsi les irrégularités de construction et donnant à l'ouvrage une allure monumentale. Au sommet de l'élévation se trouve une banchée de mortier rose qui marque le passage au couronnement

À l'angle sud-ouest de l'enceinte, sur le rocher du Matacan, subsistent les restes du couronnement antique, englobés dans des remaniements postérieurs. Cette conservation exceptionnelle a constitué la découverte la plus inattendue du projet de prospection architecturale. Le couronnement comprend un chemin de ronde, un parapet, des merlons avec des embrasures et des traverses internes pour étayer les merlons (fig. 41). Cinq traverses et trois secteurs de parapet avec leurs merlons ont été mis au jour à la suite du démontage des remaniements postérieurs. Un peu plus au nord, quatre traverses supplémentaires sont encore visibles, prises sous des réfections postérieures.

D’autres éléments du rempart antique ont fait l'objet d'une recherche dans le cadre de la prospection architecturale : les tours, les déversoirs et les portes.

À l'angle nord-ouest de l'enceinte ont été mises en évidence les traces d'arrachement d'une tour effondrée, dont l'érosion avait sapé les fondations. La restitution des emplacements éventuels d'autres tours, quoique fondée sur des observations souvent ténues plus que sur des preuves évidentes, laisse penser qu'il existait, au moins sur la façade septentrionale, une série de tours saillantes.

Au moins dix déversoirs transperçaient le rempart pour assurer l'évacuation des eaux pluviales. Ils étaient, pour la plupart, taillés dans de gros blocs de marbre de section rectangulaire, recouverts de dalles également en marbre. Du septième déversoir ne subsiste que la trace en creux de son emplacement, alors qu'un autre est constitué d'un bloc de caniveau en remploi provenant d'un bâtiment public.

À l'époque médiévale, trois portes desservaient la ville haute, mais aucun vestige d'ouvrage antique n'a été retrouvé. Il demeure cependant très probable que l'entrée principale durant l'Antiquité se situait à l'emplacement de la porte Majou d'époque médiévale.

\section{LES FOUILLES ARCHÉOLOGIQUES}

Divers sondages ponctuels, d'une superficie restreinte, furent implantés le long du tracé de l'enceinte, afin d'éclaircir l'histoire de l'occupation du sommet de la colline et de rechercher la tranchée de construction du rempart, pour obtenir des éléments fiables de datation en relation avec celle-ci. La présente contribution se bornera à une synthèse des résultats principaux acquis durant les fouilles.

Aucune couche, aucun tesson de céramique antérieurs $\mathrm{au} \mathrm{IV}^{\mathrm{e}} \mathrm{s}$. de notre ère ne furent découverts dans l'ensemble des sondages réalisés. Cette constatation rend peu plausible l'hypothèse d'un oppidum préromain sur la colline. Le sondage de 1997-1999 révéla un bâtiment en dur, construit dans la seconde moitié du IV ${ }^{\mathrm{e}} \mathrm{s}$., vraisemblablement peu avant l'édification du rempart, sur la face nord de la colline. La tranchée de construction du rempart, mise au jour sur une longueur de $5 \mathrm{~m}$ environ dans les sondages de 1994-1995 et 1997-1999, a livré un lot assez important de céramique, qui fixe la fourchette chronologique du remplissage de la tranchée à la charnière des $\mathrm{IV}^{\mathrm{e}}$ et $\mathrm{V}^{\mathrm{e}} \mathrm{s}$., probablement dans le premier tiers $d u V^{e} s$. La fortification de la colline en fit un pôle d'attraction beaucoup plus actif qu'elle ne l'était auparavant, et les traces d'occupation du $\mathrm{V}^{\mathrm{e}} \mathrm{au} \mathrm{VII}^{\mathrm{e}} \mathrm{s}$. sont beaucoup plus nombreuses que celles des époques antérieures. Dans le sondage de 1994-1995 un bâtiment en torchis avec un four en matériaux de remploi se trouvait adossé au rempart, scellé par des couches d'occupation des $\mathrm{V}^{\mathrm{e}}$ et $\mathrm{VI}^{\mathrm{e}} \mathrm{s}$. contenant un mobilier céramique important, notamment des dérivées des sigillées paléochrétiennes (DSP). Le bâtiment mis au jour dans le sondage de 1997-1999, construit vers la fin du IV ${ }^{\mathrm{e}}$ s., continua à être 
occupé tout au long des $\mathrm{V}^{\mathrm{e}}$ et $\mathrm{VI}^{\mathrm{e}} \mathrm{s}$., comme en témoigne l'importante stratigraphie de cette époque dans la pièce principale, et celui-ci fut même agrandi par l'adjonction d'une nouvelle pièce sur le côté méridional. Les couches de démolition des édifices ont livré un mobilier céramique des VII $^{\mathrm{e}}$ ou VIII $\mathrm{e}$ s.

\section{LE REMPART ET LA VILLE DE L'ANTIQUITÉ TARDIVE}

Les apports des analyses architecturales et des fouilles opérées sur le rempart antique de Convenae enrichissent nos connaissances sur la fin de la ville du Haut-Empire et sur les débuts de la ville de l'Antiquité tardive et du Moyen Âge. Il est désormais acquis que la première moitié $d u V^{e} s$. a constitué un tournant essentiel pour l'ensemble du complexe urbain de Saint-Bertrand-de-Comminges/Valcabrère. L'attribution à cette période de la mise en chantier du rempart est en parfaite cohérence avec les données disponibles (même si elles sont relativement peu nombreuses) sur la démolition des bâtiments du centre de la ville antique, située en contrebas. En effet, les observations effectuées lors des fouilles de B. Sapène et les stratigraphies analysées dans le cadre des travaux du projet collectif « Recherches pluridisciplinaires sur la cité des Convènes » (RPCC) témoignent du démontage systématique et de la récupération méthodique des matériaux pratiqués, à partir du dernier tiers du $\mathrm{IV}^{\mathrm{e}} \mathrm{s}$., sur le monument à enceinte circulaire, les thermes du forum, la demeure de Coupéré et, éventuellement, le macellum et le portique en pi. Malheureusement, la chronologie de quelques édifices importants, comme le temple du forum ou les thermes du Nord, reste mal assurée pour ces périodes, mais l'hypothèse d'un traitement identique subi à la même époque par ces édifices n'aurait rien d'invraisemblable. La présence dans le rempart d'éléments architecturaux en remploi, identiques aux colonnes, chapiteaux, corniches et caniveaux identifiés dans les édifices de la ville basse, ainsi que l'ensemble hétéroclite d'éléments en terre cuite (briques, tuiles, dalles) utilisés dans les assises de briques du parement ont déjà été soulignés. La conclusion s'impose pratiquement d'elle-même: le centre-ville antique fut démoli pour servir de carrière au rempart. Une double fonction peut être attribuée à ces fortifications. En premier lieu leur fonction défensive est évidente : installées au sommet de la colline, elles pouvaient servir de lieu de refuge et de protection pour la population et ses biens en cas d’urgence. Mais, en même temps, la qualité de la réalisation et l'allure monumentale de l'ouvrage suggèrent un rôle symbolique d'expression du statut et de l'importance de la ville, à une époque où le rempart était le monument le plus accompli des villes de l'Antiquité tardive.

La fourchette chronologique proposée pour la construction du rempart reste, en l'état actuel des données, trop large pour que soit établi un contexte historique précis pour le projet de fortification de la ville haute. Il n'en prend pas moins place dans une période de grands bouleversements politiques dans le sud-ouest de la Gaule. Élevé à la pourpre en 406, l'usurpateur breton Constantin III se fixa comme priorité de fermer le passage des Pyrénées aux barbares qui franchirent le Rhin au début de 407. Ce fut un échec et Constantin III fut vaincu et décapité à Arles en 411. Huit ans plus tard, les Wisigoths étaient établis dans le sud-ouest de la Gaule, mais l'exacte extension de leur contrôle originel reste difficile à établir. Il est possible que la Novempopulanie soit passée sous leur contrôle à partir de 419, mais, par ailleurs, aucune source ne confirme une présence wisigothique dans les Pyrénées avant le milieu du $\mathrm{V}^{\mathrm{e}} \mathrm{s}$., quand ils firent campagne en Espagne. Plutôt que de proposer une date précise, une autre façon de présenter le problème consiste à souligner que l'instabilité politique et militaire accrue dans le sud-ouest de la Gaule durant la première moitié $\mathrm{du} \mathrm{V}^{\mathrm{e}} \mathrm{s}$. créait un contexte favorable à l'établissement de fortifications. Un argument fort contre l'hypothèse qui ferait des remparts de Lugdunum une solution élaborée à la hâte face à un épisode de crise particulier est la qualité de leur construction. Le soin apporté à la planification, à la construction et à l'allure des remparts laisse penser qu'ils ne furent pas le fruit d'une nécessité pressante. Pour clore cette présentation, il faut souligner l'absence, dans la séquence stratigraphique, de toute trace du siège de 585, sauf à attribuer à cette période la surélévation de la construction observée dans le couronnement de l'ouvrage, trace qui serait à rattacher à l'activité des défenseurs de la ville, et non à celle, destructrice, des assiégeants, qui est rapportée dans le récit de Grégoire de Tours ${ }^{65}$.

65. Nous remercions vivement le professeur Robert Sablayrolles de l'université de Toulouse II-Le Mirail pour la traduction du texte anglais en français.

La publication définitive du projet paraîtra en 2006: S. Esmonde Cleary et J. Wood, Saint-Bertrand-de-Comminges-III-Le Rempart de l'Antiquité tardive de la ville haute, Bordeaux, Fédération Aquitania (coll. Études d'archéologie urbaine). 